\title{
Dezentrale Versickerung von Straßenabwässern im urbanen/innerstädtischen Raum
}

\author{
Bernhard Pucher · Roza Allabashi · Magdalena Lukavsky · Alexander Pressl · Thomas Ertl
}

Online publiziert: 3. September 2018

(c) Der/die Autor(en) 2018

\begin{abstract}
Zusammenfassung Um den Auswirkungen des Klimawandels in urbanen Gebieten wie Starkniederschläge und

\section{Decentralized infiltration of stormwater runoff from roads within urban areas} Bildung von Hitzeinseln entgegenzuwirken, sind für die Siedlungswasserwirtschaft neue Konzepte nötig. Dabei spielt der Begriff „Grüne Infrastruktur (GI)“ eine ganz zentrale Rolle. Dachbegrünung, grüne Fassade und bepflanzte Filtersysteme - sogenannte Retentionsfiltersysteme - haben ein hohes Potenzial, Extremereignissen auf lokaler Ebene entgegenzutreten. Konventionelle Entwässerungssysteme zeichnen sich durch das rasche Abführen des Oberflächenabflusses aus, sind jedoch aufgrund der steigenden Regenintensitäten und neu angeschlossenen Flächen oft nicht mehr in der Lage, alles Wasser abzuführen, was zu Überflutung und weiteren Schäden führen kann. Durch GI ist es möglich, den lokalen Wasserhaushalt wieder zu aktivieren und somit das Stadtklima positiv $\mathrm{zu}$ beeinflussen. Oberflächenabfluss aus dem Straßenraum ist je nach Nutzungsgrad unterschiedlich stark mit Kontaminanten wie z.B. Schwermetallen verunreinigt und benötigt oft eine Reinigungsstufe, bevor das Wasser in den Grundwasserkörper gelangt oder anderweitig genutzt wird. In dieser Arbeit wird die Prüfung der Eignung von Retentionsfiltersystemen für die Reinigung von Straßenabwässern erörtert. Die Entwicklung von geeigneten Filtermaterialien wird dabei mittels numerischer Simulation unterstützt, um einerseits ein Prozessverständnis solcher Anlagen zu erhalten und andererseits den Entwicklungsprozess zu unterstützen.

DI B. Pucher $(\bowtie) \cdot$ DI Dr. R. Allabashi · M. Lukavsky · DI A. Pressl · Univ.-Prof. DI Dr. T. Ertl Institut für Siedlungswasserbau, Industriewasserwirtschaft und Gewässerschutz, Universität für Bodenkultur Wien, Muthgasse 18, 1190 Wien, Österreich bernhard.pucher@boku.ac.at
Abstract In order to counteract the effects of climate change in the urban environment, namely intensive rainfall and development of heat islands, new concepts are needed. The main purpose of conventional urban drainage systems such as stormwater- or combined sewer systems is to quickly discharge the stormwater runoff into a receiving water, but due to the increasing amount of connected areas and runoff volume these static systems become more and more prone to flooding. To relief the pressure on the sewer system and more importantly reactivate the local water cycle to provide a better urban climate, green infrastructure (GI) such as green roofs, green facades as well as bioretention filters have proven to be appropriate measures on the local scale. For the infiltration of street runoff not only the volume of water but also the contamination load included has to be respected as the vast amount of infiltration water is recharging the groundwater body. In this study the treatment capabilities of bioretention filters is under investigation. For the experimental setup methods based on the Austrian standards are used and the ongoing development of appropriate filter materials is supported by numerical modelling.

\section{Einleitung}

Die fortschreitende Urbanisierung in Kombination mit den Auswirkungen des Klimawandels stellt die Stadtplanung und im Speziellen den Siedlungswasserbau vor neue Herausforderungen. Die vermehrt aufkommenden Starkregenereignisse mit Intensitäten weit über den vorgeschriebenen Bemessungsregen übersteigen die hydraulische Kapazität des Kanalsystems und können vermehrt nicht mehr zur Gänze abgeführt werden. Am Beispiel des zentralen Kanalsystems der Stadt Wien zeigt sich, dass Teile des Kanalsystems bereits ab einem 5-jährlichen Regenereignis überlastet sind (Pfannhauser 2014).

Durch die anhaltende Verbauung und Oberflächenversiegelung wird immer mehr abflusswirksame Fläche an die bestehende Infrastruktur angeschlossen. Die Auswirkungen reichen von einer Erhöhung des Mischwasserüberlaufvolumens über punktuellen Einstau im Straßenraum bis hin $\mathrm{zu}$ schwerwiegenden Überflutungen ganzer Straßenzüge sowie Schäden an Infrastruktur und Gebäuden.

Ein anderes Extrem stellt die städtische Überwärmung dar. Während die globale Mitteltemperatur im 20. Jahrhundert um knapp $0,9^{\circ} \mathrm{C}$ anstieg, liegt die Temperaturzunahme in Österreich bei $2,0^{\circ} \mathrm{C}$ (ZAMG 2018). In Wien steigt neben der Intensität auch die Anzahl an Hitzetagen (von 9,6 zwischen 1961 und 1990 auf 15,2 pro Jahr bis 2010) (Kuttler 2011; ZAMG 2012). Durch zunehmende Verdrängung der Natur durch verbaute Fläche entstehen sogenannte Urban Heat Islands (UHI) (Kuttler 2011). Diese definieren sich über den Temperaturgegensatz zwischen Stadt und Umland welcher bis $\mathrm{zu} 12^{\circ} \mathrm{C}$ betragen kann (Eliasson 2000). Ausschlaggebend für UHI ist, dass die thermische Energie der Sonneneinstrahlung in der verbauten Fläche fast zur Gänze adsorbiert wird und so ein deutlicher Anstieg der Lufttemperatur entsteht (Musco 2016).

Ganzheitlich betrachtet kann beiden Extrema durch eine Anpassung des Regenwassermanagements (RWM) in der Stadt entgegengewirkt werden. Mit Maßnahmen zur Entsiegelung durch Implementierung von Grüner Infrastruktur (GI), wie Gründächern, Fassadenbegrünung und Retentionsfiltersystemen, aber auch (teil-)durchlässige Bodenbeläge, reduziert sich der Niederschlagsabfluss, der noch bewirtschaftet werden muss (Matzinger et al. 2017). Lokal bewirtschaftetes Niederschlagswasser wird zurückgehalten, 
Tab. 1 Stoffquellen und Stoffemissionen durch Kfz Verkehr und Dächer

\begin{tabular}{ll}
\hline $\begin{array}{l}\text { Stoffquelle } \\
\text { Straßen }\end{array}$ & Stoffe \\
\hline Abgase & $\begin{array}{l}\text { Stickoxide, Kohlendioxid, Ruß, Kohlenstoff, Blei, Schwefel, Chlor, Magnesium, Natrium, Kupfer, Zink, } \\
\text { Kohlenwasserstoffe, PAK, Phenole, PCDD/PCDF }\end{array}$ \\
\hline Fahrbahn Beläge (Abrieb) & Silizium, Calcium, Magnesium, Chrom, Nickel, Bitumen \\
\hline Reifen (Abrieb) & $\begin{array}{l}\text { Chrom, Zink, PAK } \\
\text { Kohlenstoff, Eisen, Magnesium, Barium, Silizium, Schwefel, Titan, Chrom, Vanadium, Nickel, Zink, } \\
\text { Kremsbeläge (Abrieb) }\end{array}$ \\
\hline Kenzin, Katalysatoren & Blei, Nickel, Kobalt, Platin, Palladium, Rhodium, PAK, MTBE \\
\hline Tropfverluste & Öle, Kraftstoffe, Bremsflüssigkeit, Frostschutzmittel \\
\hline Verdampfungsverluste & Kohlenwasserstoffe \\
\hline Korrosion & Eisen, Kupfer, Cadmium, Zink \\
\hline Dächer & Kupfer, Zink, Blei, Zinn \\
\hline Schwermetallinstallationen, Dachbleche, Fassaden & Pestizide (z. B. Atrazin) \\
\hline Atmosphärische Auswaschung & Pestizide (z. B. Mecoprop, Diuron) \\
\hline Flachdachisolationen & \\
\hline
\end{tabular}

gespeichert und steht dadurch Pflanzen und somit auch der Verdunstung zur Verfügung, überschüssiges Wasser trägt durch Tiefenversickerung der Grundwasserneubildung bei. Dadurch lässt sich einerseits eine Entlastung des bestehenden Kanalsystems und andererseits durch den Verdunstungsprozess eine Kühlung der Umgebung erzielen (WSSE 2015). Auf Grundlage der Notwendigkeit eines lokalen Wasserkreislaufes wird beispielsweise in Deutschland ein Anhang zum Wasserhaushaltsgesetz erstellt, welcher den Erhalt des natürlichen Wasserhaushalts bei entwässerungstechnischen Neuerschließungen vorschreibt (DWA-A 102 2016).

Mit der Förderung des lokalen Wasserhaushalts gehen jedoch auch Gefahren einher. Bezogen auf die Quantität ist eine unnatürliche Erhöhung der Grundwasserneubildung in Gebieten mit schon erhöhten Grundwasserständen $\mathrm{zu}$ vermeiden. Qualitativ muss berücksichtigt werden, dass Niederschlagsabfluss im urbanen Raum ein variables Verschmutzungspotenzial enthält, welches eine Gefahr für Oberflächen- und Grundwasser darstellt (DWA-A 138 2005). Im Zuge des Planungsprozesses lässt sich die Qualität des Oberflächenabflusses am besten über die Nutzung der jeweiligen Fläche ableiten (Simperler et al. 2018).

Niederschlagsabfluss von Dächern und Straßen enthält neben Nährstoffen wie Stickstoff und Phosphor auch Feststoffe (abfiltrierbare Stoffe - AFS) sowie organische und anorganische Schadstoffe wie Schwermetalle, polycyclische aromatische Kohlenwasserstoffe (PAK) und Mineralöl-Kohlenwasserstof- fe $(\mathrm{MKW})$. Diese entstehen mehrheitlich aus Abgasnebenprodukten, Reifenund Karosserie- sowie Fahrbahnverschleiß. Die Höhe der jeweiligen Belastung hängt von der durchschnittlichen täglichen Verkehrsbelastung (DTV) ab und kann somit stark variieren (Welker und Dittmer 2005; in Schmitt et al. 2010).

Die wichtigsten Ursachen für Verunreinigungen und deren Quellen sind in Tab. 1 zusammengefasst (Boller 2003; Helmreich 2005). Die relativen Beiträge der unterschiedlichen, von Fahrbahnen abgeleiteten Verunreinigungen (z.B. das Verhältnis von Kupfer zu Stickstoff zu abfiltrierbaren Stoffen) hängen stark von der Landnutzung rund um die Verkehrsflächen ab (Choe et al. 2002).

Die oben beschriebenen Verunreinigungen im Oberflächenabfluss stellen ein Potenzial zur Gefährdung des Grundwassers bei dessen Versickerung dar, daher müssen die gesetzlichen Grundlagen des Gewässerschutzes beachtet werden.

Der Gewässerschutz wird auf europäischer Ebene durch die Wasserrahmenrichtlinie (RL 2000/60/EG idF 2009/31/EG) sowie die Grundwasserrichtlinie (RL 2006/118/EG idF 2014/80/EU) vorgegeben. Die Umsetzung auf nationaler Ebene wird durch das Wasserrechtsgesetz (WRG $1959 \mathrm{idF}$ 2013) realisiert. Für den Schutz von Oberflächengewässern gilt es, den guten Zustand quantitativ sowie qualitativ zu erreichen und nicht $\mathrm{zu}$ verschlechtern. Für das Grundwasser wird die Einhaltung bzw. Erreichung des guten chemischen und mengenmäßigen $\mathrm{Zu}$ stands sowie ein Verschlechterungsverbot des guten Zustands vorgeschrieben.
Für das Grundwasser ist der gute chemische Zustand über Schwellenwerte für Schadstoffe in der Qualitätszielverordnung Chemie Grundwasser (QZV Chemie GW, BGBl. II 98/2010 idF BGBl. II 461/2010) vorgegeben welche Einbringungsverbote sowie -beschränkungen festgelegt.

Für die Nutzung von Filtermaterialien beinhaltet die ÖNORM B 2506 Teil 2 und 3 (ÖNORM B2506-2 2012; ÖNORM B2506-3 2016) die nachzuweisenden Prüfverfahren (Haile und Fürhacker 2017). Hierbei ist anzumerken; dass diese Norm generell für technische Filtermaterialien entwickelt wurde.

\section{Projekt SAVE}

Basierend auf modellgestützten Klimasimulationen zeigen Matzinger et al. (2017), dass für das Stadtklima die Implementierung von Baumscheiben und Teichen am effektivsten Hitzestress reduzieren kann. Um zu verhindern, dass die Abwässer den Wurzelraum von Bäumen gefährden, sind in Wien die sogenannten Baumscheiben vom Straßenkörper entkoppelt. Dabei geht nötiges Wasser für die Bewässerung der angrenzenden GI verloren.

Gemeinsam mit der Stadt Wien wird im Projekt SAVE (Straßen-Abwasserlösungen für Vegetation und Entwässerungssysteme) nach interdisziplinären Lösungen für das urbane RWM gesucht. Dabei umspannt das Projekt die Entwicklung und Untersuchung von geeigneten Filtermaterialien für die Retention, Behandlung und Infiltration von Straßenabwässern vom Labor bis hin zur Implementierung von mehreren Pilotsystemen im Stadtgebiet und de- 
ren Monitoring über eine Periode von ca. 3 Jahren. Der Einsatz dieser GI steht dabei auch im Kontext der sogenannten Baumscheiben, da hier die Nutzung des Infiltrationswassers für die Bewässerung der Bäume im Stadtgebiet einen weiteren Nutzungsbereich darstellt.

\subsection{Entwicklung und Optimierung von Filtermaterialien}

Die Zusammensetzung des Filtermaterials spielt eine entscheidende Rolle in Retentionsfiltersystemen in Hinblick auf $\mathrm{pH}$-Wert, gesättigte hydraulische Leitfähigkeit, Infiltrationsrate, Kationenaustauschkapazität sowie Partikelund Schadstoffrückhalt. Diese Faktoren sind äußerst wichtig für den Einsatz von Retentionsfiltern zur Behandlung des Oberflächenabflusses aus dem Straßenraum und Lebensraum für die Vegetation. Der Partikeleintrag in Form von Sand, Schluff und Ton sowie organischen Bestandteilen aus dem Oberflächenabfluss hat in weiterer Folge einen signifikanten Einfluss auf die Mobilität und den Rückhalt von Schwermetallen. Gleiches gilt für die im Winterdienst eingesetzten Taumittel. Durch die Beigabe von technischen Materialien $\mathrm{zu}$ den Retentionsfiltersystemen wie etwa Sand und Schotter wird eine stabile Struktur gewährleistet, um die notwendige Infiltration und Retention zu gewährleisten (Gülbaz et al. 2015). Zusätzlich sollte je nach geplanter Bepflanzung ein den Anforderungen entsprechender Oberboden verwendet werden (Søberg et al. 2017).

Die Entwicklung von Filtermaterialien für den Einsatz als GI mit dem Hauptziel der Wasserretention und der Entfernung von im Straßenabfluss ent-

Tab. 2 Überblick der Anforderungen an das Filtermaterial

\begin{tabular}{|c|c|}
\hline Eigenschaft & Anforderung \\
\hline Infiltrationsrate (Ki) & $\geq 1 \times 10^{-5} \mathrm{~m} / \mathrm{s}$ \\
\hline Partikelretention & $>80 \%$ \\
\hline \multirow[t]{3}{*}{ Schwermetallrückhalt } & $\begin{array}{l}\text { Cu-Entfernungsrate } \\
>80 \%\end{array}$ \\
\hline & $\begin{array}{l}\text { Zn-Entfernungsrate } \\
>50 \%\end{array}$ \\
\hline & $\begin{array}{l}\text { Pb-Konzentration } \\
<9 \mu \mathrm{g} / \mathrm{l}\end{array}$ \\
\hline $\begin{array}{l}\text { Änderung der } \\
\text { Infiltrationsrate }\end{array}$ & $<50 \%$ \\
\hline \multirow{2}{*}{$\begin{array}{l}\text { Remobilisierung der } \\
\text { Schwermetalle durch } \\
\text { Salz }\end{array}$} & $\begin{array}{l}\text { Cu-Konzentration } \\
<50 \mu \mathrm{g} / \mathrm{l}\end{array}$ \\
\hline & $\begin{array}{l}\text { Zn-Konzentration } \\
<500 \mu \mathrm{g} / \mathrm{l}\end{array}$ \\
\hline
\end{tabular}

haltenen Schadstoffen basiert grundlegend auf der ÖNORM B 2506-3 (2016), die die Anforderungen an Filtermaterialien zur Reinigung von Niederschlagsabflüssen von Dächern und befestigten Flächen beschreibt. Die genannte Norm bezieht sich jedoch nur auf technische Filtermaterialien, weshalb für naturnahe Infiltrationssysteme eine adaptierte Methodik entwickelt wurde. Die Evaluierung erfolgt im Labor mittels Säulenversuchen. Das Flächenverhältnis von Filterfläche zu entwässernder Fläche wurde mit 1:15 angenommen und entspricht den allgemeinen Gegebenheiten des urbanen Raumes (Beinlich 2016). Ein Überblick der Anforderungen an den geprüften Filtermaterialien ist in Tab. 2 aufgelistet.

Es wurden mit dieser Methode elf unterschiedliche Mischungen untersucht und anschließend evaluiert. Für die Evaluierung wurden alle gemessenen Parameter laut Tab. 2 herangezogen und mit einem gewichteten Index versehen. Für den Grundwasserschutz relevante Parameter werden als sehr wichtig eingestuft und weisen einen höheren Gewichtungsindex auf. Wenn eine Schadstoffkonzentration die Anforderung der ÖNORM B2506-3 (2016) nicht entspricht, wird das Material als „nicht geeignet“ eingestuft. Im Gegensatz dazu wurden Materialien, die die Anforderung „Änderung der Infiltrationsrate" nicht erfüllten $(<50 \%)$ nicht ausgeschlossen, sondern mit einer niedrigeren Einstufung versehen (Beinlich 2016). Fünf dieser Mischungen haben sich als geeignet erwiesen und wurden für die Weiterverwendung als Filtermaterial in Retentionsfiltersystemen empfohlen.

Bei der endgültigen Auswahl der Materialien für die Feldimplementierung wurden zusätzlich auch Anforderungen des spezifischen Standorts berücksichtigt, wie z.B. Infiltrationsfähigkeit der Grundschicht, verfügbarer Raum, Art des technischen Systems, Qualität und Menge des Straßenabflusses sowie die Intensität des Winterdienstes berücksichtigt. Dementsprechend wurde für die Feldversuche im Rahmen des Projektes SAVE für jeden konkreten Standort eine individuelle Lösung vorgeschlagen.

\subsection{Problematik Winterdienst}

Neben der ganzjährigen Belastung im Straßenraum wird während des Winterdienstes eine weitere Belastung in
Form von Taumittel hinzugefügt. Die umfangreiche Ausbringung von Streusalz kann zu Schäden an Fahrzeugkarosserien und Betonbauwerken führen. Bezogen auf die Umwelt wird das Salz gelöst im Oberflächenabfluss der GI zugeführt. Zu $90 \%$ wird Natriumchlorid $(\mathrm{NaCl})$ verwendet. Während $\mathrm{Na}$ in der ungesättigten Zone zurückgehalten wird, wird $\mathrm{Cl}$ ausgewaschen und gelangt so folglich in den Grundwasserkörper.

Hohe Konzentrationen an $\mathrm{NaCl}$ können sich im Boden auf unterschiedliche Weise negativ auf physikalische und chemische Eigenschaften der Böden wie Verschlämmung, Verdichtung, Nährstoffauswaschung und die Bodenlösung auswirken. Dadurch wird die Wasserbeweglichkeit gehemmt, die Durchlüftung verringert und die freigesetzten Nährstoffe stehen den Pflanzen nicht mehr zur Gänze zur Verfügung. Durch die Verkrustung der Bodenstruktur wird die Versorgung der Wurzeln weiter erschwert. Mit steigender Salzkonzentration steigt das osmotische Potenzial der Bodenlösung. Dies bewirkt eine Verringerung des Wurzelturgors und führt $\mathrm{zu}$ physiologisch trockenen Böden.

Durch ein Überangebot von $\mathrm{Na}$ in der Bodenlösung werden durch Ionentausch Kationen wie $\mathrm{K}$, $\mathrm{Ca}$ und $\mathrm{Mg}$ von den Bodenkolloiden desorbiert und $\mathrm{Na}$ adsorbiert, wodurch deren Gehalt in der Bodenlösung steigt, bis sich ein neuer Gleichgewichtszustand einstellt. Die desorbierten Kationen (K, Ca, Mg) können leicht verlagert und bei durchlässigen Böden auch ausgewaschen werden.

Chloridionen unterliegen nicht einem Ionentausch und werden leicht mit dem Sickerwasser verlagert und ausgewaschen. Durch erhöhte Infiltration (Regen- oder Tauwetter) kann das Chlorid innerhalb weniger Tage aus dem Boden ausgewaschen werden und gelangt so in den Grundwasserkörper. Allerdings können Restkonzentrationen auch noch Jahrzehnte in der ungesättigten Zone verbleiben. Der ChloridTransport in der ungesättigten Zone hängt von bodenphysikalischen $\mathrm{Pa}$ rametern, der Vegetation, Tiefe und Schwankung des Grundwasserspiegels ab. Bei stark verdichteten und wenig durchlässigen Böden wird der Austrag aus dem Wurzelraum beispielsweise verlangsamt und behindert. Der Transport von Salz durch das Grundwasser kann an Stellen, wo der Grundwasserspiegel relativ flach ist, zu hohen Chloridkonzentrationen in oberflächenna- 
hen Böden führen. Obwohl der Streusalzabfluss im Boden verdünnt wird, können in flachen Grundwasserbereichen in städtischen Gebieten immer noch Cl-Konzentrationen von Hunderten bis zu mehreren tausend mg. $\mathrm{L}^{-1}$ auftreten (Foos 2003; Panno et al. 1999). Die Chlorid Konzentration nimmt mit der Tiefe des Bodens (z.B. Pitt et al. 1996) zu. Für unterschiedliche Geologie/Hydrogeologie sollten verschiedene Ansätze für die Berechnung der Chlorid-Konzentration und den Transport in der ungesättigten Bodenzone verwendet werden.

Im Wiener Stadtgebiet liegen die Chlorid-Werte derzeit noch unter dem Grenzwert, aber es gibt einen deutlich erkennbaren ansteigenden Trend mit Einzelwerten über dem Schwellenwert (Pfannhauser 2014). Die Analyse der Daten hat gezeigt, dass ein unmittelbarer Einfluss der Salzstreuung an einigen Messstellen mit großer Wahrscheinlichkeit gegeben ist. Wresowar und Sieghardt (2000) zeigen in ihrer Literaturzusammenstellung, dass etwa $40 \%$ des ausgebrachten Streusalzes mit den Fahrbahnabflüssen in Straßenrandböden und zwischen 5 bis $25 \%$ der ausgebrachten Streumenge mit der Verkehrsgischt aufgewirbelt und verfrachtet werden.

Um festzustellen, wie sich unterschiedliche Taumittel auf den Schwermetallrückhalt und die hydraulische Leistung in Retentionsfiltersystemen auswirken, wurden vier Versuchsanlagen im Technikum des Instituts für Siedlungswasserbau und Gewässerschutz der Universität für Bodenkultur Wien installiert (Lukavsky et al. 2018). Jede verfügt über eine Fläche von $0,23 \mathrm{~m}^{2}$ und eine Höhe von $0,75 \mathrm{~m}$. Die Beschickung mit Schwermetallkonzentrationen und partikulären Stoffen (nach ÖNORM B 2506-3 (2016)) war für alle Anlagen gleich. Drei dieser Anlagen wurden in weiterer Folge zusätzlich mit Taumittel beschickt. Zum Einsatz kamen $\mathrm{NaCl}$, Calcium-Magnesium-Acetat (CMA) und Kaliumformiat (KF). Die vierte Anlage blieb ohne Taumittelbeschickung und diente als Referenz. Um Änderungen des hydraulischen Verhaltens $\mathrm{zu}$ beobachten, wurde vor und nach jedem Beschickungsschritt die Ablaufkurve jeder Versuchsanlage für eine definierte Beschickung mit Wasser gemessen. Diese Daten sind auch für die in Abschn. 3. beschriebene Modellierung wichtig.

\section{Modellierung von \\ Retentionsfiltersystemen}

Die mathematische Beschreibung des Wasser- und Stofftransports in Filtersystemen gibt Aufschluss über eben jene vorherrschenden Prozesse und kann zur Abschätzung der Retentionskapazität, des Verdunstungspotenzials und des Schadstoffrückhalts genutzt werden.

Damit die modellbasierte Beschreibung eine gute Abbildung der Realität zulässt, ist es unumgänglich, schon während der Versuchsdurchführung etwaige zusätzliche Parameter zu bestimmen und somit den Versuchsablauf zu erweitern.

Für die Simulation wird das Softwarepaket HYDRUS 2D/3D (Šimůnek et al. 2016) verwendet. HYDRUS beschreibt die 2D-Bewegung von Wasser, Wärme und gelösten Stoffen in der gesättigten sowie ungesättigten Bodenzone. Zur Beschreibung der Wasserbewegung in der Bodenmatrix löst HYDRUS die Richards-Gleichung (Richards 1931), welche auch einen Term für Aufnahme von Bodenwasser über die Wurzeln berücksichtigt. Stoff- sowie Wärmetransport werden durch die Konvektions-Dispersions-Gleichung in der flüssigen sowie der Gasphase realisiert (Šimůnek et al. 2016).

Die bodenphysikalische Beschreibung des Filtermaterials erfolgt durch das bodenphysikalische Modell von van Genuchten/Mualem (van Genuchten 1980; Mualem 1976). Die benötigten Parameter sind in Tab. 2 aufgelistet. Die Prüfung der Filtermaterialien nach ÖNORM B 2506-3 (2016) liefert zwei Parameter, welche die grundlegenden Eigenschaften des Materials bestimmen, einerseits die gesättigte hydraulische Leitfähigkeit und andererseits den gesättigten Wassergehalt unter der Annahme, dass dieser zirka der Porosität entspricht (Ramos et al. 2006). Die weiteren Parameter werden im Zuge der Kalibrierung gefittet, d.h. durch inverse Modellierung bestimmt. Hierfür werden Messdaten wie z.B. eine Ablaufkurve aus einem Säulenversuch oder Messungen des Bodenwasserhalts bei bekannter Beschickung benötigt. Bei der inversen Modellierung werden die gesuchten bodenphysikalischen Parameter so lange verändert, bis eine Zielfunktion ihr Minimum erreicht (Šimůnek et al. 1999). Die so erhaltenen Parameter zuzüglich zu den gemessen sind als beschreibend für das jeweilige Filtermaterial anzusehen.

Die Messung der benötigten Ablaufkurven wurden einerseits im Zuge der Prüfung gemäß ÖNORM B 25063 (2016) an den Säulen bzw. im Zuge der Versuche an den Versuchsanlagen durchgeführt.

Mittels numerischer Simulation soll die Änderung der hydraulischen Leistungsfähigkeit anhand der beschriebenen bodenphysikalischen Parameter dargestellt werden. Dies erfolgt durch Bestimmung der neuen gesättigten hydraulischen Leitfähigkeit über die inverse Modellierung mittels der bestimmten Abflusskurven am Ende der Versuchsdurchführung an den Versuchsanlagen. Dieser erste Schritt dient als Ausgangspunkt für die Abschätzung der Nutzungsdauer der eingesetzten Filtermaterialen sowie die Beschreibung und Methodenentwicklung für weitere Untersuchungen von Retentionsfiltersystemen.

\section{Resultate}

\subsection{Resultate aus den Versuchsanlagen}

Der Schwermetallrückhalt in den Versuchsanlagen unter Einfluss verschiedener Taumittel ist in Abb. 1 dargestellt. Insgesamt zeigt die untersuchte Filtermischung eine hohe Rückhalterate für die Schwermetalle auch unter Einfluss von Taumitteln. Der Schwermetallrückhalt unter Einfluss von $\mathrm{NaCl}$ liegt bei allen fünf Metallen über $90 \%$. Der Rückhalt unter Einfluss von CMA bei $\mathrm{Pb}, \mathrm{Cr}$ und $\mathrm{Cu}$ liegt bei über $90 \%$ und bei Ni und $\mathrm{Zn}$ bei über $80 \%$. Unter Einfluss von KF liegen die Werte für $\mathrm{Pb}$, $\mathrm{Cu}$ und $\mathrm{Zn}$ über $90 \%$ und für $\mathrm{Cr}$ und $\mathrm{Ni}$ über $80 \%$. Der Referenzdurchlauf weist für alle Schwermetalle einen Rückhalt über $90 \%$ auf. Auf den Rückhalt von $\mathrm{Pb}$ und $\mathrm{Cu}$ können keine Auswirkungen von Taumitteln beobachtet werden. Bei den drei anderen Schwermetallen zeigen die Taumittel eine Verringerung des Rückhalts in der Reihenfolge $\mathrm{Ni}>\mathrm{Cr}>\mathrm{Zn}$. Vergleichbare Ergebnisse wurden bei den Säulenversuchen zur Remobilisierung von Schwermetallen erzielt und stehen im Einklang mit der Literatur (Bäckström et al. 2004; Helmreich et al. 2017). Eine Erhöhung der Niund Zn-Mobilität ist durch die Zugabe des Taumittel CMA zu erkennen, während $\mathrm{KF}$ die Mobilität von $\mathrm{Ni}$ und $\mathrm{Cr} \mathrm{zu}$ beeinflussen scheint. Aus der Untersuchung der Abflusskonzentrationen ist 


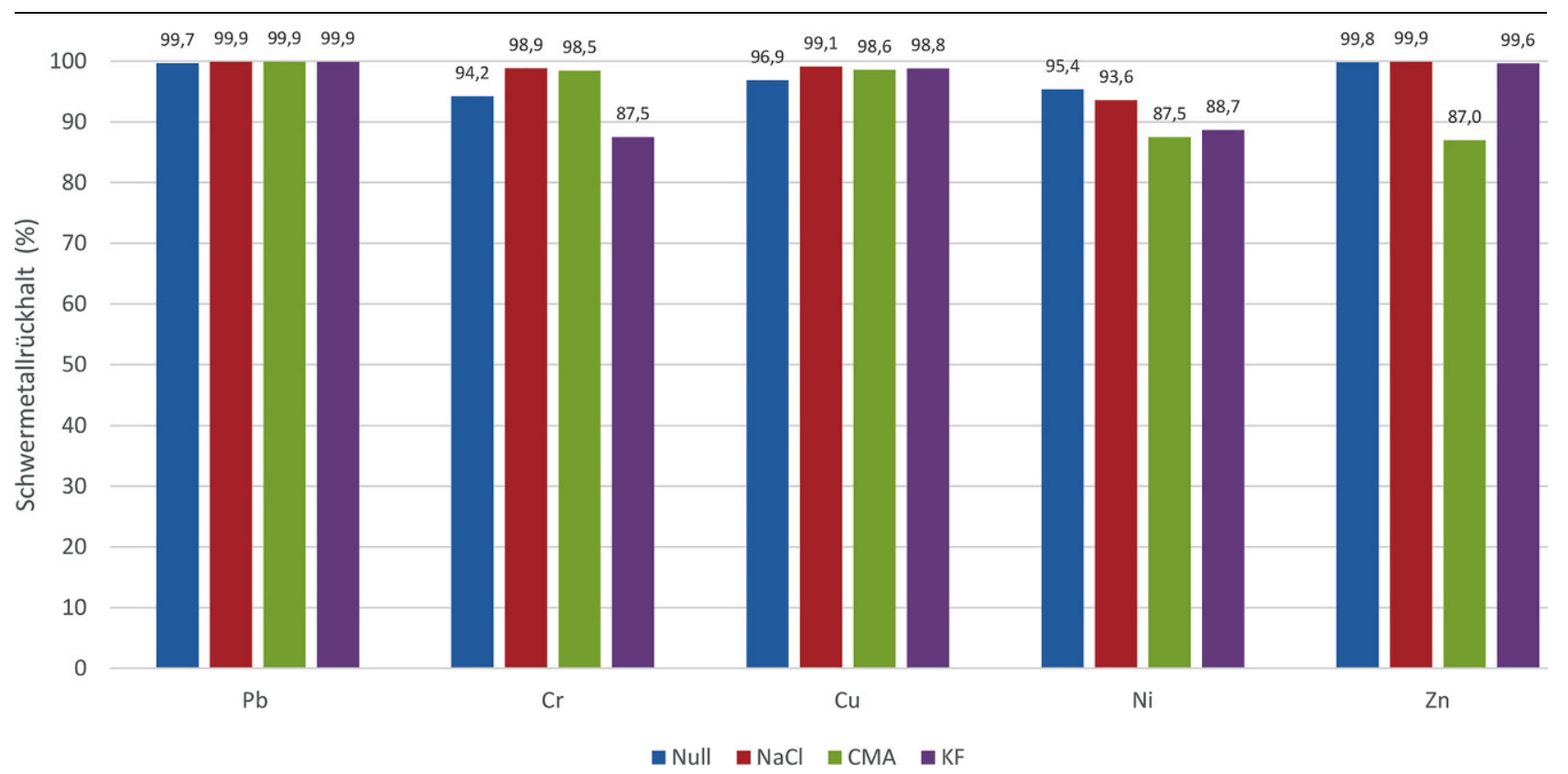

Abb. 1 Schwermetallrückhalt unter Einfluss der getesteten Taumittel (Lukavsky et al. 2018)

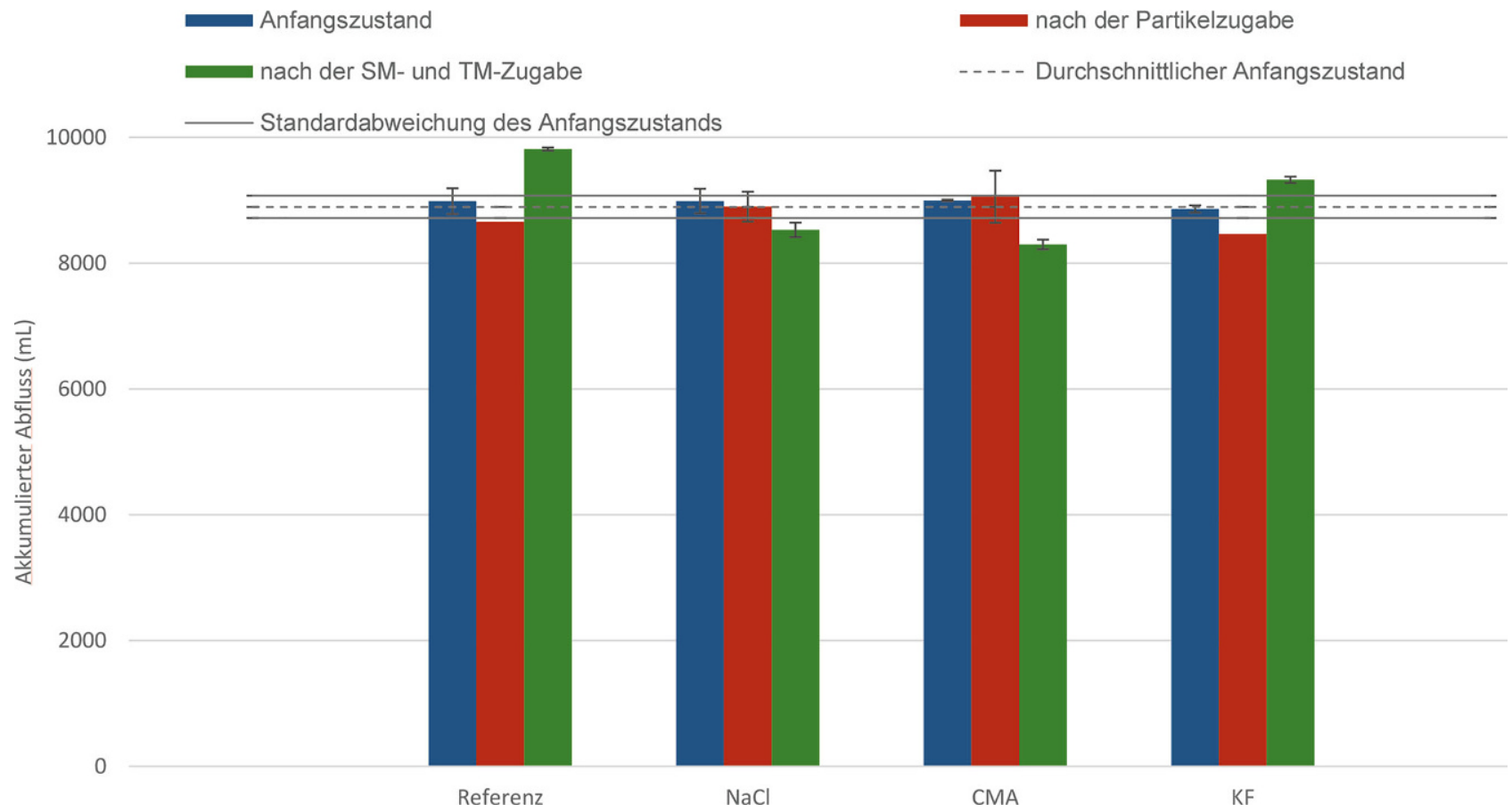

Abb. 2 Akkumulierter Abfluss nach 30 min mit vorangegangener Beschickung von 15L Wasser (SM Schwermetalle; TM Taumittel) (Lukavsky et al. 2018)

zu schließen, dass trotz der Erhöhung der Mobilität bei Ni, Cr und Zn lediglich bei Ni vereinzelt Überschreitungen des Grenzwerts lt. Qualitätszielverordnung Chemie Grundwasser festzustellen waren.

Die Messungen der Abflusskurven unterliegen der Annahme, dass höhere Abflussraten auf eine höhere hydrau- lische Leitfähigkeit rückschließen lassen. Abb. 2 zeigt, dass demnach die Partikelzugabe wenig Einfluss auf die Hydraulik nimmt und die Taumittelschwermetalllösung eine signifikante Änderung des hydraulischen Verhaltens verursacht. Durch $\mathrm{NaCl}$ und CMA scheint die Durchlässigkeit sich zu verringern, durch $\mathrm{KF}$ und durch die reine
Schwermetalllösung zu erhöhen. Die Fehlerbalken in Abb. 2 stellen die Standardabweichungen der letzten zwei gemessenen Abflusskurven bei vorhandener Datenlage dar.

Die Ergebnisse der Laborversuche nach der Säulen-Methode, wie in der ÖNORM B 2506-3 (2016) beschrieben, zeigen eine deutliche Verringerung der 
Tab. 3 Beschreibung der Parameter des bodenphysikalischen Modells nach van Genuchten/Mualem

$\begin{array}{llll}\text { Parameter } & \text { Beschreibung } & \text { Bestimmung } \\ \frac{\Theta \mathrm{r}}{\left(\mathrm{cm}^{3} \cdot \mathrm{cm}^{-3}\right)} & \text { Restwasser welches in den Poren gegen die Gravitation gehalten wird und nicht Pflanzenverfügbar ist } & \text { Inverse Modellierung } \\ \frac{\Theta \mathrm{s}}{\left(\mathrm{cm}^{3} \cdot \mathrm{cm}^{-3}\right)} & \text { Gesättigter Wassergehalt, kann weitestgehend mit der gemessenen Porosität angenommen werden } & \text { ÖNORM B 2506-3 (2016) } \\ \frac{\alpha}{\left(\mathrm{cm}^{-1}\right)} & \text { Formparameter der Wasserspannungs-Wassergehaltskurve } & \text { Inverse Modellierung } \\ \frac{\mathrm{n}}{(-)} & & \text { Inverse Modellierung } \\ \frac{\mathrm{ks}}{\left(\mathrm{cm} \cdot \mathrm{min}^{-1}\right)} & \text { Gesättigte hydraulische Leitfähigkeit } & \text { ÖNORM B2506-3 } \\ \frac{1}{(-)} & \text { Tortuosität, beschreibt die Gewundenheit der Transportwege im Porenraum } & \text { Inverse Modellierung }\end{array}$
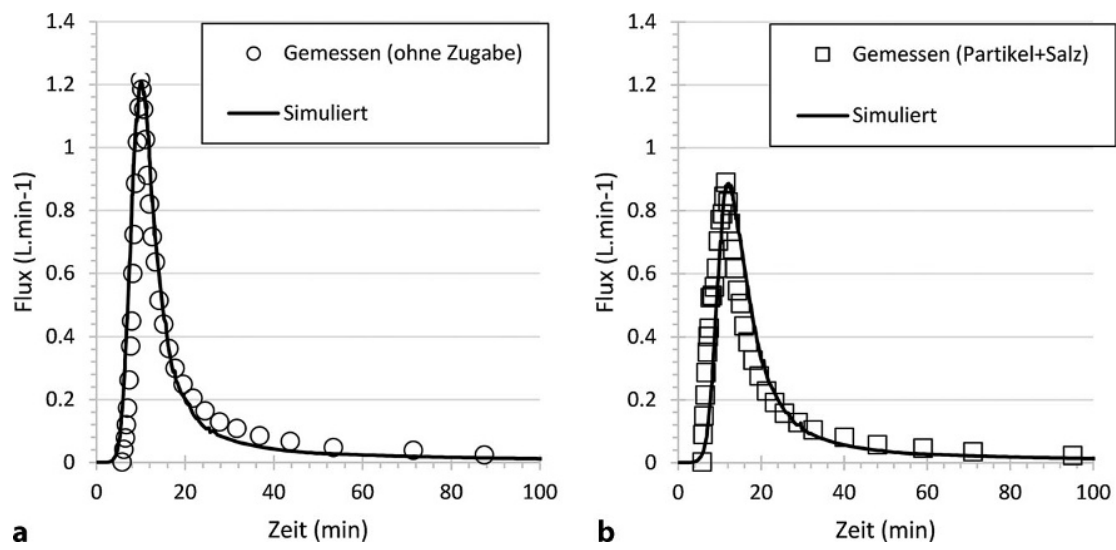

Abb. 3 Gegenüberstellung der gemessenen und simulierten Abflusskurve für die Versuchsanlage 2, welche mit $\mathrm{NaCl}$ beschickt wurde (a zeigt die Daten und Simulationsergebnisse vor der Partikel- und Salzzugabe, b die Daten danach)

gesättigten hydraulischen Leitfähigkeit nach der Partikelzugabe und eine Steigerung nach Zugabe aller drei Taumittel. Die Rückschlüsse aus den Abflusskurven sind daher nicht vergleichbar mit der Messung der gesättigten hydraulischen Leitfähigkeit. Die geringe Standardabweichung des Anfangszustands (Abb. 2) deutet die Reproduzierbarkeit der Methode an. Die Interpretation der Daten in Hinsicht auf hydraulische Prozesse ist jedoch mit Unsicherheit behaftet, jedoch ist ein genereller Einfluss auf die hydraulische Leistung durch den Einsatz von Taumittel feststellbar.

\subsection{Erste Resultate der Modellierung von Infiltrationssystemen}

Basierend auf den hydraulischen Versuchen wurde ein erstes Modell für die Versuchsanlage 2 (Beschickung mit $\mathrm{NaCl})$ erstellt. In Abb. 3 ist das Ergebnis der Kalibrierung mittels inverser Simulation dargestellt. Für den Ausgangszustand wurden die Parameter $\Theta r, \alpha, n$ und l gefittet (siehe Tab. 3). Mittels der gemessenen Abflusskurve am Ende der Versuche wurde in weiterer Folge nur die gesättigte hydraulische Leitfähigkeit gefittet. Die Veränderung der Abflusskurven wird durch diese Methode äußerst genau beschrieben (Abb. 3).

\section{Schlussfolgerungen}

Für die Wasserwirtschaft stellt die Aufrechterhaltung des natürlichen Wasserkreislaufes ein wesentliches Ziel dar (ÖWAV 2015). Je nach betrachteter Fläche kann ein zentrales Kanalsystem diesen Grundsatz einhalten, in Bezug auf den lokalen Wasserhaushalt im Stadtgebiet jedoch nicht. Daher stehen das urbane Regenwassermanagement (RWM) und alle beteiligten Instanzen vor neuen Herausforderungen, welche ein Umdenken zu den bisherigen Lösungen fordert. Abhilfe schafft hier auch nicht der alleinige Einsatz neuartiger Technologien, sondern eine ganzheitliche Betrachtung auf der Systemebene, hier die Ebene des urbanen Raums.

Die in diesem Beitrag beschriebene Nachweisführung zur Einhaltung der Wasserqualität für die Versickerung von Straßenabwässern folgt einerseits der Methodik der ÖNORM B 2506-3 (2016), kann aber von dieser nicht zur Gänze erfasst werden, da es sich bei den besprochen Infiltrationssystemen nicht um technische Filter handelt, sondern um naturnahe, bepflanzte Systeme. Das ÖWAV-Regelblatt 45 (ÖWAV 2015) sieht hierfür die Prüfung auf Eignung zum Rückhalt der anfallenden Schadstoffe vor. Aus den beschriebenen Ergebnissen lässt sich diese Eignung des zum Einsatz kommenden Filtermaterials in Hinblick auf Teile der ÖNORM B 25063 (2016) sowie des Gewässerschutzes (QZV Chemie GW, BGBl. II 98/2010 idF BGBl. II 461/2010; WRG 1959 idF 2013) ableiten.

Die Prüfung auf Eignung (ÖWAV 2015) soll zukünftig auch von der numerischen Modellierung unterstützt werden. Die erfolgreiche Kalibrierung der bodenphysikalischen Parameter sowie das Fitting der Änderung der gesättigten hydraulischen Leitfähigkeit zeigt die Tauglichkeit der numerischen Simulation für die Beschreibung von Retentionsfiltersystemen. Die bis jetzt erhaltenen Ergebnisse sind von qualitativer Natur. Um die Änderung der hydraulischen Leitfähigkeit quantitativ ausdrücken zu können, ist in den nächsten Schritten eine Validierung notwendig.

Danksagung Die Autorinnen und Autoren danken der Stadt Wien für die Finanzierung des SAVE-Projekts.

Funding Open access funding provided by University of Natural Resources and Life Sciences Vienna (BOKU).

Open Access Dieser Artikel wird unter der Creative Commons Namensnennung 4.0 International Lizenz (http:// creativecommons.org/licenses/by/4. $0 /$ deed.de) veröffentlicht, welche die Nutzung, Vervielfältigung, Bearbeitung, Verbreitung und Wiedergabe in jeglichem Medium und Format erlaubt, sofern Sie den/die ursprünglichen $\mathrm{Au}$ tor(en) und die Quelle ordnungsgemäß nennen, einen Link zur Creative Commons Lizenz beifügen und angeben, ob Änderungen vorgenommen wurden. 


\section{Literatur}

Bäckström, M., Karlsson, S., Bäckman, L., Folkeson, L, Lind, B. (2004): Mobilisation of Heavy Metals by Deicing Salts in a Roadside Environment. Water Research 38(3), 720-732.

Beinlich, L. (2016): Evaluation of filter materials for urban stormwater management - green infraestructure in the city of Vienna. Vienna, Austria.

Boller, M. (2003): Nachhaltige Regenwasserentsorgung auf dem Weg in die Praxis. EAWAG news 57, 25-28.

Choe, J. S., Bang, K. W., Lee, J. H. (2002): Characterization of surface runoff in urban areas. Water Science and Technology45(9), 249-254.

DWA (Deutsche Vereinigung für Wasserwirtschat) (2005): Arbeitsblatt DWA-A 138 Planung. Bau und Betrieb von Anlagen zur Versickerung von Niederschlagswasser.

DWA (Deutsche Vereinigung für Wasserwirtschat) (2016): Arbeitsblatt DWA-A 102 Grundsätze zur Bewirtschaftung und Behandlung von Regenwetterabflüssen zur Einleitung in Oberflächengewässer - Entwurf.

Eliasson, I. (2000): The use of climate knowledge in urban planning. Landscape and Urban Planning 48, 31-44.

Foos, A. (2003): Spatial distribution of road salt contamination of natural springs and seeps. Environmental Geology 44 (1), 14-19.

van Genuchten, M. T, (1980): A Closed-form Equation for Predicting the Hydraulic Conductivity of Unsaturated Soils. Soil Science Society of America Journal 44(5), 892-898.

Gülbaz, S., Kazezyllmaz-Alhan, C. M., \& Copty, N. K. (2015): Evaluation of Heavy Metal Removal Capacity of Bioretention Systems. Water, Air \& Soil Pollution, 226(11)

Haile, T. M., Fürhacker, M. (2017): Filtermaterialprüfung: Anwendung der ÖNORM B 2506 Teil 3 für das hochrangige Straßennetz. Österreichische Wasser- und Abfallwirtschaft, 69(11-12), 495-502.

Helmreich, B., Hilliges, R., Schriewer, A., Athanasiadis, K. (2005): Schadstoffe aus Niederschlagsabflüssen und deren Bewertung. In 33. Abwassertechnisches Seminar: Niederschlagswasserbehandlung in urban Gebieten 185 37-48.

Helmreich, B., Huber, M., Rempe, L. M., Drewes, J. E. (2017): Entwicklung einer zweistufigen Behandlungsanlage für Verkehrsflächenabflüsse unter Berücksichtigung urbaner und kommunaler Anforderungen. In: Urbanes Niederschlagsmanagement im Spannungsfeld zwischen zentralen und dezentralen Maßnahmen, U1-U12 Graz: Institut für Siedlungswasserwirtschaft und Landschaftswasserbau, Technische Universität Graz.

Kuttler, W. (2011): Klimawandel im urbanen Bereich. Teil 2, Maßnahmen. Environmental Sciences Europe, Vol. 23, Issue 21

Lukavsky, M., Pucher, B., Haile, T. M., Ertl, T. Allabashi, R. (2018): Einfluss von Taumitteln auf den Schwermetallrückhalt und das hydraulische Verhalten von Substraten in Versickerungssystemen. [Poster]. In: Schmitt, G. (Hrsg.): Aqua Urbanica 2018 - Regenwasser in urbanen Räumen aqua urbanica trifft Regenwasser Tage 2018.; ISBN: 978-3-95974-086-9.

Matzinger, A., Riechel, M., Remy, C., Schwarzmüller, H., Rouault, P., Schmidt, M., Offermann, M., Strehl, C., Nickel, D., Sieker, H., Pallasch, M., Köhler, M., Kaiser, D., Möller, C. Büter, B., Leßmann, D., von Tils, R., Säumel, I., Pille, L., Winkler, A., Bartel, H., Heise, S. Heinzmann, B., Joswig, K., Rehfeld-Klein, M. Reichmann, B. (2017): Zielorientierte Planung von Maßnahmen der Regenwasserbewirtschaftung - Ergebnisse des Projektes KURAS. Berlin. Mualem, Y. (1976): A new model for predicting the hydraulic conductivity of unsaturated porous media. Water Resources Research 12(3), 513-522.

Musco, F. (Ed). (2016): Counteracting Urban $\mathrm{He}-$ at Island Effects in a Global Climate Change Scenario. Springer International Publishing.

ÖNORM B 2506-2 (2012): Regenwasser-Sickeranlagen für Abläufe von Dachflächen und befestigten Flächen, Teil 2: Qualitative Anforderungen an das zu versickernde Regenwasser sowie Anforderungen an Bemessung, Bau und Betrieb von Reinigungsanlagen.

ÖNORM B 2506-3 (2016): Regenwasser-Sickeranlagen für Abläufe von Dachflächen und befestigten Flächen, Teil 3: Filtermaterialien: Anforderungen und Prüfmethoden, Österreichisches Normungsinstitut, Wien, Österreich.

ÖWAV (2015): Oberflächenentwässerung durch Versickerung in den Untergrund, ÖWAV - Regelblatt 45, Österreichischer Wasser- und Abfallwirtschaftsverband, Wien.

Panno, S. V., Nuzzo, V. A., Cartwright, K., Hensel, B. R., Krapac, I. G. (1999): Impact of urban development on the chemical composition of ground water in a fen-wetland complex. Wetlands 19, 236-245.

Pfannhauser, G. (2014). Straßenentwässerung und Regenwassermanagement der Stadt Wien: Themenbereich Dezentral Regenwasserbewirtschaftung Einsparungspotential im Straßenbau. Studie zur Erstellung eines verbindlichen Leitfadens.

Pitt, R., Clark, S., Parmer, K., Field R. (1996): Groundwater Contamination from Stormwater Infiltration, Ann Arbor Press, Inc. Chelsea, Michigan.

QZV (2010): Qualitätszielverordnung Chemie Grundwasser, Pub. L. No. BGBl. II Nr. 98/2010, Anlage 1.

Ramos,T. B., Gonc Alves, M. C.,Martins, J. C.,van Genuchten, M. Th., Pires, F. P. (2006): Estimation of soil hydraulic properties from numerical inversion of tension disk infiltrometer data. Vadose Zone J. 5(2), 684-696.

Richards, L. A. (1931): Capillary conduction of liquids in porous mediums. Physics 1, 318-333.
Schmitt, T. G., Welker, A., Dierschke, M., Uhl, M., Maus, Ch., Remmler, F. (2010): Entwicklung von Prüfverfahren für Anlagen zur dezentralen Niederschlagswasserbehandlung im Trennverfahren, Abschlussbericht über ein Entwicklungsprojekt, gefördert unter dem Az: 26840-23 von der Deutschen Bundesstiftung Umwelt, Jul 2010.

Simperler, L., Himmelbauer, P., Stöglehner, G., Ertl, T. (2018): Siedlungswasserwirtschaftliche Strukturtypen und ihre Potentiale für die dezentrale Bewirtschaftung von Niederschlagswasser. Österreichische Wasser- und Abfallwirtschaft, Österreichische Wasser- und Abfallwirtschaft, 70 (11-12), in diesem Heft.

Søberg, L. C., Viklander, M., \& Blecken, G.-T. (2017): Do salt and low temperature impair metal treatment in stormwater bioretention cells with or without a submerged zone? Science of The Total Environment 579, 1588-1599.

Welker, A., Dittmer, U. (2005): „Belastung von Verkehrsflächenabflüssen mit Schwermetallen und PAK - Ergebnisse einer Literaturstudie“. gwf-Wasser/Abwasser 146(4), 320-332.

Wresowar M., Sieghardt, M. (2000): Studie über die Auswirkung stickstoffhältiger Auftaumittel Auswirkungen auf Boden und Bewuchs Vergleich mit herkömmlichen Auftaumitteln, BOKU Wien im Auftrag der Magistratsabteilung 22 der Stadt Wien.

WSSE (2015): Dynamische Anpassung regionaler Planungs- und Entwicklungsprozesse an die Auswirkungen des Klimawandels in der Emscher-Lippe-Region (Ruhrgebiet), gefördert 2009-2015 durch das Bundesministerium für Bildung und Forschung (BMBF) im Rahmen des KLIMZUG-Programms.

Zentralanstalt für Meteorologie und Geodynamik (ZAMG) (2012): Ö Hitzetage werden immer häufiger. Online im Internet: URL: https://www. zamg.ac.at/cms/de/klima/news/hitzetagewerden-immer-haeufiger [Abruf 31.07.2018].

Zentralanstalt für Meteorologie und Geodynamik (ZAMG) (2018) Informationsportal Klimawandel. Online im Internet: URL: https:/ / www. zamg.ac.at/cms/de/klima/informationsportalklimawandel/standpunkt/klimavergangenheit/ neoklima/lufttemperatur [Abruf 01.08.2018] Šimůnek, J., O. Wendroth, and M. Th. van Genuchten (1999): Estimating unsaturated soil hydraulic properties from laboratory tension disc infiltrometer experiments. Water Resour. Res. 35 , 2965-2979.

Šimůnek, J., van Genuchten, M. T., Šejna, M. (2016): Recent developments and applications of the HYDRUS Computer Software Packages. Vadose Zone Journal 15(7), DOI: 10.2136/vzj2007.0077. 